\title{
VIII. On the cyanides of the metals, and their combinations with cyanide of potassium. Part I. Cyanide of gold
}

\section{Messr Charles F.O. Glassford \& Messr James Napier}

To cite this article: Messr Charles F.O. Glassford \& Messr James Napier (1844) VIII. On the cyanides of the metals, and their combinations with cyanide of potassium. Part I. Cyanide of gold, Philosophical Magazine Series 3, 25:163, 56-66, DOI: 10.1080/14786444408644929

To link to this article: http://dx.doi.org/10.1080/14786444408644929

冓 Published online: 30 Apr 2009.

Submit your article to this journal $₫$

Џ Article views: 1

Q View related articles $\llbracket$ 
"Difar Sir,

"121 Camden Road Villas, Camden Town, March 7, 1842.

"I have to apologize for not writing sooner, but I have had a smart attack of asthma, which is now gone off.

"Graham's experiments are very ingenious, but I do not see that they can be applied to explain the diffusion of gases as laid down by Dalton, and now universally admitted. I consider gases and steam (so long as it is steam) follow exactly the same laws, the only difference being that steam liquefies at an accessible temperature and pressure, whereas the gases liquefy at temperatures and pressures that are almost inaccessible. I view all gaseous bodies as elastic fluids that act upon one another and upon other bodies by the laws of elastic fluids; and this seems to be sufficient to account for all the physical properties discovered by experiment. It also gets rid of the only exceptionable part of Dalton's theory, namely his hypothesis that one gas is inelastic to all other gases, which, although it be intelligible in words, is incomprehensible by the understanding.

"I do not apprehend that your manner of explaining Graham's experiments is liable to objection.

"To Thos. S. Thomson, Esq., "Believe me to be, yours, \&c., 5 Bury St., St. James's." "James Ivorx."

VIII. On the Cyanides of the Metals, and their Combinations with Cyanide of Potassium. Part I. Cyanide of Gold. By Messrs. Charles F. O. Glassford and James Napier*. 1. THE various compounds which cyanogen forms with the metals constitute one of the most interesting class of bodies which the science of chemistry can produce; and the rapid progress of electro-metallurgy, with the almost universal use of these cyanides in the practice of this art, adds a double interest to the study of these compounds with the al. kaline cyanides. Indeed, we might have expected that long before this some definite information would have been published upon the nature, constitution and easy preparation of these salts, as yet however, so far as we are aware, nothing complete has appeared. With a view to remedy this, and to facilitate a full investigation of the subject, we have drawn up the following observations, the result of extensive practical operations in the art of electro-metallurgy, which we beg to present to the Chemical Society, in the hope that our results, in connexion with those of others who may be labouring in the

* Communicated by the Chemical Scciety; having been read February $19,1844$. 
same field, may lead to a more precise knowledge of their character and constitution.

2. In systematic works on chemistry reference is made to this subject in a very general way. The sum of such information may be thus expressed:- " The soluble cyanides of the alkaline metals, potassium, sodium, \&c., dissolve the insoluble cyanides of the metals proper, forming double salts, which are generally crystallizable:" the probable constitution of only a very few of these salts are given.

3. From the researches of L. Gmelin upon the metallocyanurets, which were published many years ago, it would appear that there are two classes of compounds in which other metals are found to replace the iron in the yellow and red prussiate of potash, and to form indeed metallo-cyanurets and sesquicyanurets of potassium, \&c. There seems however to be a singular indefiniteness on this subject in chemical works; for 'Turner, in his 'Elements,' 5th edition, p. 779, says, that "L. Gmelin has obtained a double cyanuret of zinc and potassium, in which the zinc acts as iron in the ferro-cyanurets." He also says, "that a cobalto-cyanuret of potassium, and similar double cyanurets of nickel and cobalt, may be prepared in the same manner as the preceding salt;" and further, " that Gmelin procured the cobalto-cyanuret in yellow crystals, similar in composition and form to ferro-cyanuret of potassium." The salts here described are evidently analogous to the yellow prussiate of potassa, or ferro-cyanuret of potassium; but on referring to Professor Graham's 'System,' we find there described salts of cobalt, chromium and manganese, not analogous to yellow prussiate of potash, but similar in symbolic constitution to the red prussiate of potash, that is, metallo-sesquicyanurets having a supposed salt radical in which these metals replace iron to the formation of cobalti-, chromi-, and mangani-cyanogen; no mention is made whatever of the existence of compounds similar to yellow prussiate of potash, or of a cobalto-, chromo-, and mangano-cyanogen.

On turning to Berzelius's Traité, tome ii. p. 115, we find a description of the same zinco-cyanuret of potassium, or, as he names it, cyanuri zincoso-potassique, analogous in composition to the yellow prussiate of potassa, in which zinc is found to replace iron. He says, "The existence of this salt, of which the discovery is due to L. Gmelin, leads us to presume that zinc has a series of double cyanurets, like iron, nickel, \&c." Here also Berzelius corroborates the statement made by Dr. Turner, and speaks of these compounds as analogous to the yellow prussiate of potassa, or as metallo-cyanurets. Turner mentions (p. 780) that Gmelin's observations upon the nickel, copper and zinco-cyanureis have been confirmed by a late 
pupil of his, Mr. F. Rodgers, and his brother; on referring to their Memoir, published in the Philosophical Magazine of February 1834, we find a different statement from what Dr. Turner makes; they say, "L. Gmelin prepared a double cyanuret of cobalt and potassium, analogous to the red cyanuret of iron and potassium." These different statements of apparently the same thing must have arisen either from the want of proper care in naming the salts, so as to indicate to which class they belong, or to the want of sufficient evidence respecting their constitution. We may render this still more evident by the following statements. Berzelius mentions a cyanure platinoso-potassique, or platino-cyanuret of potassium, but on referring to its alleged constitution, we do not find, as its name indicates, a compound of platina similar to the ferrocyanuret of potassium, but a compound totally different from either the yellow or red prussiate of potassa ; the same salt is described in Graham's ' Elements,' p. 991; and there also it is called the platino-cyanide of potassium. The acid described in some chemical works as the platino-cyanic acid, does not resemble in constitution the ferro-cyanic acid, as its name indicates. If, therefore, there be a necessity for distinguishing the acid formed by the supposed salt radical ferri-cyanogen with 3 eqs. of hydrogen, from that formed by ferro-cyanogen with 2 eqs. of hydrogen, so then there is an equal necessity for distinguishing a metallo-cyanogen, which combines with 2 eqs. of hydrogen, from a metallo-cyanogen, which combines with 1 eq. of hydrogen : for the same reason also platino- and other cyanides of potassium should receive appellations which indicate their constitution, and not such as by analogy lead us to infer a totally different constitution. These remarks tend to show us the necessity of carefully re-examining the nomenclature of this apparently complex subject, and the scrupulous care with which we ought to classify and name these compounds, so as neither to misrepresent their constitution, nor to leave the possibility of their being ascribed to classes to which they do not belong.

4. We deem these observations necessary for the purpose of alluding to a memoir by Mons. A. Meillet, which appeared in the Journal de Pharmacie et de Chemie for June last, and which was translated into the Philosophical Magazine for August, entitled, "On some new combinations of Cyanogen." The author says, "The peculiar manner in which cyanogen acts towards iron, by forming two very stable acids with it, leads to the supposition that it is not the only metal with which cyanogen is capable of combining. In fact, some German chemists, and Gmelin among others, have discovered three new compounds, which are platino-cyanogen, cobalto- 
cyanogen, and chromo-cyanogen, and afterwards the hydrogenated acids analogous to ferro-hydrocyanic acid, and several other metallic salts." Here again we have more evidence of the singular confusion with which this subject has become invested. If we are to accept these names for what they express, and as yet we have no reason to do otherwise, we must infer that these metallo-cyanogens are analogous to the ferrocyanogen; but this is not the case, for we have just shown that platino-cyanide of potassium is not similar in constitution to the ferro-cyanide of potassium. Meillet then proceeds to say, "that the processes employed by these chemists were somewhat complicated, and they had not continued their experiments ;" the method which he employed, he says, "is simple, and there may be procured by it a great number of perfectly definite compounds." The remainder of the paper is taken up with a brief description of the characteristics of these salts; among others he mentions the auro-, the argentoand the cupro-cyanides of potassium, classifying these under their supposed salt radicles, as auro-cyanogen, argento-cyanogen, and cupro-cyanogen. It is in the consideration of these various metallic combinations with cyanogen, that we have made the following investigations. Before commencing the detail of these, we must offer a few observations upon the cyanide of potassium as usually prepared, and as employed by Meillet in his experiments.

5. The cyanide of potassium which is employed by us in our daily operations is prepared according to the process first pointed out by Messrs. F. and E. Rodgers, now generally known by the appellation "Liebig's Process;" from what particular cause it came to be so named we know not, unless it may have been that the publication of this process by Professor Liebig occurred at a time when the attention of practical and scientific men were more particularly drawn towards an attentive consideration of this compound, from its important applications in chemical analyses, and its extensive employment in the new art of electro-metallurgy. To show, however, that this process was known long before this illustrious chemist pointed it out, we may be allowed to quote the following passage from the memoir of Messrs. Rodgers, already mentioned as having been published in the Philosophical Magazine for February 1834. After detailing the various processes then known, and others which they had discovered, they describe this process as follows:- "Cyanuret of potassium may be prepared by exposing a mixture of anhydrous carbonate of potash and anhydrous ferro-cyanuret of potassium to a moderate red heat, in a covered porcelain crucible, for about twenty minutes." "The proportions employed should 
be nearly 1 eq. of anhydrous carbonate of potash to 1 eq. of the anhydrous ferro-cyanuret of potassium. The process is very productive, for the weight of the cyanuret of potassium obtained by this process is much greater than the weight of the cyanuret obtained by heating ferro-cyanuret of potassium alone, owing to the cyanogen of the cyanuret of iron uniting with the potassium contained in the carbonate of potash; and, moreover, the process occupies less time. Some carburet of iron always remains in the crucible."

6. Cyanide of potassium may be obtained pure for experimental investigation in the Jaboratory by the use of absolute alcohol; but where hundred weights are prepared and used at a time this is not admissible. As employed in the arts it contains many impurities: these are chiefly cyanate, carbonate, muriate and sulphate of potash, silica, and yellow prussiate of potash. The average amount of these impurities is 35 per cent., often as high as 50 per cent. It contains much silica when prepared, as is usually directed, in earthenware crucibles; and when the exact proportions of the yellow prussiate of potash or carbonate of potash are not employed; when these are not perfectly anhydrous, salts of ammonia are generated; and when commercial carbonate of potash is used, muriate and sulphate of potash. Even when the materials are employed chemically pure and perfectly anhydrous, it contains 20 per cent. of cyanate of potash ; this, according to Professor Liebig, is of little consequence in the ordinary operations of testing, but in respect to analytical inquiry, and to electrometallurgical operations, we find it of the greatest importance. As however we shall have opportunities of pointing these out in detail, we only allude to them here in a general way.

7. When nitrate of silver is added to solution of cyanide of potassium, just so long as the precipitate which is at first formed is redissolved, we obtain the rehole of the cyanide of potassium in union with the silver, none of the other salts present, although amounting to a large per-centage, taking any part in the action. Upon this law we have founded a method of estimating the exact quantity of pure cyanide of potassium in any sample; as, however, the principle upon which this depends can be better explained when the nature and constitution of the cyanide salt of silver is described, we shall return to it when treating of that metal.

8. As when one eq. of chloride of gold combines with one eq. of chloride of potassium, a double salt, called chloride of gold and potassium, is formed, so shall we call the union of one eq. of cyanide of gold with one eq. of cyanide of potassium the "cyanide of gold and potassium," and apply similar names to the other metallic salts having a like constitution. The 
terms double-cyanides, bicyanides, \&c., which are usually given to such compounds, we consider neither necessary nor sufficiently expressive.

9. The equivalent numbers employed are those given in Dr. Turner's 'System,' namely,-Cyanogen 26.39 , potassium $39 \cdot 15$, cyanide of potassium $65^{\circ} 54$, gold $199 \cdot 20$, cyanide of gold $225 \cdot 59$.

10. Cyanide of Gold.-Little is said in any systematic treatise of the cyanide of gold. Berzelius, almost the only chemist who mentions this compound, says, "That this salt presents itself under the form of a pale yellow substance, insoluble in water, which is obtained by precipitating the chloride of gold with cyanide of potassium, and gives as its composition the formula $\mathrm{Au}, \mathrm{Cy}_{3}$." Kane also mentions this pale yellow compound, and gives it the same composition. It may readily be obtained by decomposing the chloride of gold with cyanide of potassium, but both solutions must be saturated, and care must be taken not to add an excess of cyanide of potassium ; the product however by this process is very small compared to the quantity of gold employed, and it is necessary to proceed as follows to obtain the whole of the gold as cyanide :Saturated solutions are to be used, and a slight excess of cyanide of potassium added, so as to redissolve the cyanide of gold; the whole is to be evaporated to perfect dryness, treated with nitric or muriatic acids to decompose the cyanide of potassium, again evaporated to dryness, and gently heated so as to expel the excess of acid, when cold water is to be added, and the whole thrown upon a filter and thoroughly washed. Obtained in this way however it generally contains a portion of silica, particularly if the cyanide of potassium has been prepared in earthenware crucibles; and to obtain the cyanide pure we have recourse to other methods. The best process is to decompose the cyanide of gold and potassium. The salt is to be dissolved in a small portion of distilled water, nitric or muriatic acid added, and the liquid brought to a boiling heat, the cyanide of gold then precipitates; but it is necessary to expel the excess of acid by evaporation before the whole of the cyanide is obtained; the dry mass is then treated with water, thrown upon a filter and well washed.

The cyanide of gold obtained by the last method is of a beautiful canary yellow colour, which is not affected by exposure to light, or by the action of acids: it is an exceedingly fine powder, and readily passes through a single filter; it is insoluble in caustic potash and water; is slightly soluble in nitric, muriatic, and sulphuric acids when recently precipitated; is soluble in ammonia, hyposulphite of soda, and readily so in cyanide of potassium; when fused at a red 
heat with carbonate of potash, it is entirely decomposed, and the gold may be obtained in a bright metallic button.

11. The cyanide obtained by the decomposition of the double salt by acids was examined in this way and the following results obtained :-

$$
\begin{aligned}
& 10 \text { grs. yielded } 8.82 \text { grs. of gold }=88.20 \text { per cent. } \\
& 15 \text { grs. } \quad \ldots \quad 13.23 \text { grs. } \quad \ldots=88.20 \quad \ldots \\
& 7 \text { grs. } \ldots \quad 6.17 \text { grs. } \ldots=88 \cdot 14 \quad \ldots
\end{aligned}
$$

These results approximate so very closely to the calculated result, viz. 88.30 per cent., that we are at once led to the conclusion that it is a true proto-cyanide, the constitution of which is represented by $\mathrm{Au}+\mathrm{Cy}$.

A cyanide of the constitution represented by Berzelius and Kane would contain only 71.56 per cent.

The cyanide of gold obtained by the first process is generally contaminated, as already mentioned, with silica and a small portion of iron, obtained from the cyanide of potassium employed : these impurities will vary according to the state of the cyanide used. Before these facts were ascertained the analyses of the cyanide of gold yielded the following very variable results, -85.00 per cent., 85.50 per cent., 85.57 per cent.; this last sample, when thoroughly washed with water, carefully dried and examined, gave still only $86^{\circ} 00$ per cent.

12. Cyanide of Gold and Potassium.-This salt, which is that now so extensively employed in gilding by the electric force, may be obtained by various processes:-1. By dissolving the cyanide of gold, prepared by the first process just given, in cyanide of potassium. 2. By dissolving oxide of gold in cyanide of potassium. The oxide of gold is prepared for this purpose by digesting calcined magnesia in nitro-muriate of gold, as is well known; as it is very apt to retain a portion of magnesia as usually prepared, it is better to boil the oxide so obtained in strong nitric acid, to filter through close muslin or calico, allowing the green nitrate solution to fall into a large quantity of distilled water. The oxide of gold is thus obtained quite pure, and as a very bulky reddish-brown precipitate, which when dried contracts, and becomes of a pale brownish-yellow colour: it dissolves readily in cyanide of potassium, with the assistance of a little heat, or by digestion, and constitutes the salt in question. 3. It may also be obtained, as already mentioned, by adding a solution of cyanide of potassium to terchloride of gold until the solution becomes colourless. As the reactions which take place with these solutions are exceedingly interesting, and calculated to add evidence to the existence of a hitherto unobserved law, which will be fully brought out when treating of the double silver 
salt, and to explain similar reactions with platina and other metals, we will endeavour to follow them.

13. When a saturated solution of cyanide of potassium is added, in small portions, to a cold and strong solution of nitromuriate of gold, there is at first a violent effervescence, the solution becomes slightly turbid, and then quite transparent. The reaction may be explained as follows :-

The first effect is the neutralization of the excess of nitric and muriatic acids contained in the nitro-muriate of gold. This results at the expense of the cyanate of potash and cyanide of potassium.

Thus $-2 \mathrm{KCy}+\mathrm{KO}, \mathrm{CyO}+2 \mathrm{HCl}+\mathrm{NO}_{5}+3 \mathrm{HO}$, producing or represented thus :

$$
2 \mathrm{KCl}+\mathrm{KONO}_{5}+2 \mathrm{CO}_{2}+\mathrm{NH}_{3}+2 \mathrm{Cy} \mathrm{H} \text {, }
$$

$$
\begin{array}{r}
2 \mathrm{KCy}\left\{\begin{array}{l}
2 \mathrm{Cy} \\
2 \mathrm{~K} \\
2 \mathrm{H}
\end{array} 2 \mathrm{CyH}\right. \\
2 \mathrm{Cl} \longrightarrow 2 \mathrm{KCl}
\end{array}
$$

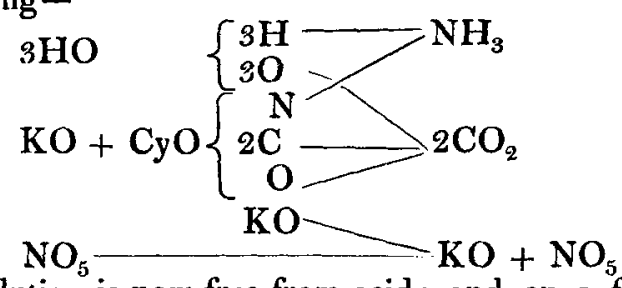

The solution is now free from acid; and on a further addition of cyanide of potassium it immediately, by the assistance of water, reacts upon the terchloride of gold. This will be evident from the following formulæ,

producing

$$
2 \mathrm{AuCl}_{3}+8 \mathrm{KCy}+8 \mathrm{HO} \text {, }
$$

$$
2(\mathrm{AuCy}+\mathrm{KCy})+6 \mathrm{KCl}+2\left(\mathrm{NH}_{3}+\mathrm{CO}_{2}\right)+2 \mathrm{HCy}
$$

or it may be represented thus :-

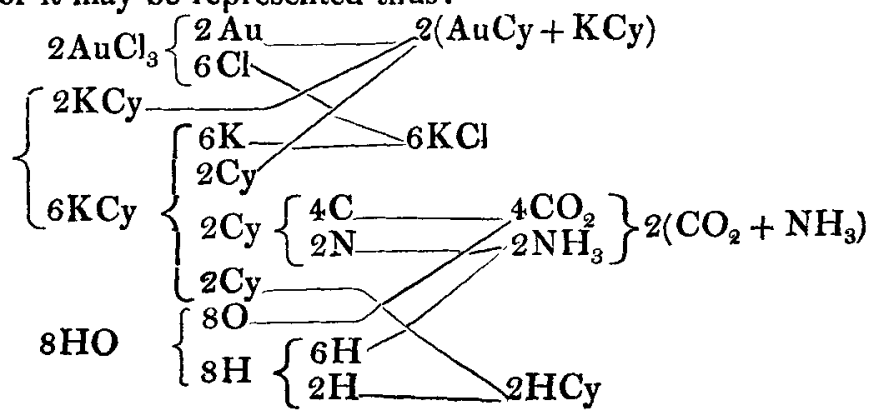


When, however, the solution of nitro-muriate of gold is recently prepared, saturated and hot, a portion of the ammonia formed reacts upon a portion of the gold, forming aurate of ammonia, which precipitates along with the cyanide of gold; on the addition of cyanide of potassium the ammonia is liberated and the double salt of gold and potassium is produced.

14. The following process which we have employed is beautifully calculated for obtaining a large quantity of salt in a very few hours, and at comparatively little trouble.

A solution of cyanide of potassium is prepared, which contains about six times as much cyanide as the quantity of gold wished to be dissolved. The cyanide may be dissolved in twice, thrice or four times its weight of distilled water and carefully filtered to separate any insoluble matters with which the cyanide is usually contaminated; this solution is then to be gently and carefully raised to a temperature of $100^{\circ} \mathrm{F}$. in an earthenware or glass vessel. Two plates of gold are placed in the solution at the opposite sides of the vessel, and these connected by means of copper wires to the poles of a small galvanic battery, composed of two or three pairs of zinc and copper plates, and excited with dilute acid. As soon as the circuit is completed the operation commences, gold is dissolved from that plate connected with the copper end of the battery, passes into the solution, and is only partly deposited upon the opposite plate, whilst a gentle flow of gas is evolved from the negative pole. The solution of cyanide of potassium rapidly consumes the gold which is dissolved at the positive pole and becomes a double salt of cyanide of gold and potassium. The gold which deposits upon the negative pole is in minute grains, occasionally quite brown, and is beautifully crystalline. The operation is much facilitated by having the positive pole or plate of gold several times larger than the opposite plate. At first the amount of gold deposited is very small compared with that dissolved from the positive pole, the solution retaining the greater proportion; but after some hours, as the solution becomes saturated, the amount deposited increases until there is a perfect balance. The solution is now to be filtered and carefully evaporated; it yields an abundant crop of crystals of the double salt. In this way half an ounce of gold may be dissolved in a few hours, and nearly an ounce of the salt obtained.

15. The salt obtained by either of these processes presents the same general appearance and constitution. It crystallizes in very minute octahedrons, which cluster together so as to form long, jagged, irregular prisms, which again group round a centre and form starlike masses; they possess a peculiar bitter 
and metallic taste. They are perfectly colourless and transparent, but seem opake from being composed of numerous small crystals. They are soluble in four times their weight of cold water, and in less than their own weight of boiling water, in the proportion of 5 to 4 . They are very sparingly dissolved by alcohol, and their solubility is not increased by boiling. When alcohol is added to a warm saturated solution of the crystals, the salt falls in fine white brilliant shining scales, which are quite opake. Their solution does not stain the hands by the action of light, neither is the solution affected by exposure to light. When nitric, muriatic, or sulphuric acid is poured over the crystals, they are not affected at first, but by standing some time they are quite decomposed, and cyanide of gold results. The cyanide of gold occasionally precipitates in minute shining crystals from the nitric solution. 'Their solution is not immediately effected in the cold by these acids, but a boiling temperature decomposes them, the yellow cyanide of gold precipitating. Tartaric, oxalic and acetic acids effect the same decomposition when boiled. The nitric, muriatic and sulphuric acids added to a cold saturated solution precipitate the salt undecomposed. The following metallic solutions were added to a solution of the salt, and the results were as follows:-

Nitrate of copper . . a white precipitate of the mixed cyanides.

Sulphate of zinc...

Nitrate of silver...

Nitrate of mercury . a yellow-white precipitate $\quad \ldots$ Acetate of lead ... a white precipitate

Protosulph. of iron - a white precipitate of the mixed cyanides, which changes to a purplish-blue on the addition of nitric acid.

Muriate of man- . small crystals appear on the sides of the ganese ...... glass on standing.

Muriate of tin .... a slight opacity, increasing to a precipitate on standing.

When exposed to a red heat with their own weight of carbonate of potash, the whole of the gold is obtained in the metallic state as a bright button. The carefully dried salt was treated in this way and the following results obtained:-

16. A salt prepared with oxide of gold and cyanide of potassium yielded the following results:-

7 grs. yielded 4.67 gold $=66.71$ per cent.

5 grs. $\quad \ldots \quad 3.33 \ldots=66.66 \quad \ldots$

$10 \mathrm{grs} . \quad \ldots \quad 6.63 \quad \ldots=66.30 \quad \ldots$

The salt prepared by the battery process was also examined; it gave the following results :-

Phil. Mag. S. 3. Vol. 25. No. 163. July 1844. 
20 grs. yielded 13.32 gold $=66.60$ per cent.

20 grs. $\quad \ldots \quad 13.25 \ldots=66.25 \quad \ldots$

A sample of the former salt was carefully recrystallized, and placed for some time under a receiver over oil of vitriol; it gave the following result:-

20 grs. yielded 13.35 of gold $=66.75$ per cent.

The mean of these results is . . . $\overline{66 \cdot 54} \ldots$

10 grs. of the salt were treated with nitric acid, and, the acid being completely expelled by evaporation, they were carefully collected and dried, yielded 8.85 grs. of cyanide of gold $=88.50$ per cent.

The calculated amount of gold and of cyanide of gold contained in a salt consisting of single equivalents of cyanide of gold, cyanide of potassium and water, is of gold 66.37 , and of cyanide of gold $88 \cdot 30$.

Our results approximate so closely to the calculated result, that we consider ourselves warranted in giving the formula

$$
\mathrm{AuCy}+\mathrm{KCy}+\mathrm{HO} \text {. }
$$

17. In conclusion we may add, that from these results we have no hesitation in saying, that the salt described by Meillet as prepared by saturating chloride of gold with cyanide of potassium is not the auro-cyanide of potassium, but the cyanide of gold and potassium as described above.

IX. On the Cyanides of the Metals, and their Combinations roith Cyanide of Potassium. Part II. Cyanide of Silver. By Messis. Charles F. O. Glassford andJames Napier*. THE compound that is formed by the union of cyanogen with silver has been long known, and its composition ascertained to be 1 equivalent of silver with 1 equivalent of cyanogen. The method of preparing this salt, as given in most chemical works, is by ardding hydrocyanic acid to the nitrate or any other soluble salt of silver, when the cyanide of silver falls as a white precipitate. This method we have found, on the large scale, to be both disagreeable and tedious, and have therefore adopted the following method for its preparation. To a neutral solution of nitrate of silver is added cyanide of potassium so long as a precipitate is formed. No hydrocyanic acid is given off, the precipitate being washed from the nitrate of potash may be used for any purpose required. If the cyanide of potassium, however, contains any cyanate or carbonate, they form impurities in the precipitated cyanide of silver; these can be separated by digesting the

* Communicated by the Chemical Society; having been read March 4, 1844. 